\title{
Clinical and left ventricular function outcomes up to five years after dynamic cardiomyoplasty
}

\begin{abstract}
Improvement in congestive heart failure and left ventricular function after dynamic cardiomyoplasty has been reported in patients with severe cardiomyopathies, but the long-term effects of this procedure remain unclear. In this investigation 31 patients undergoing cardiomyoplasty for treatment of idiopathic dilated cardiomyopathy were annually investigated with radionuclide scintigraphy, Doppler echocardiography, and right-sided heart catheterization. They were in New York Heart Association functional class III or IV before the operation. No hospital deaths occurred, but one patient with progressive heart failure required urgent heart transplantation 42 days after cardiomyoplasty. The other patients were followed up from 6 to 70 months (mean 25.6 months) and 12 patients died at late follow-up. Actuarial survivals were $86 \%$ at 1 year, $61.4 \%$ at 2 years, and $42.5 \%$ at 3 to 5 years of follow-up. Multivariate analysis of factors influencing outcome showed that long-term survival was significantly affected by preoperative functional class and pulmonary vascular resistance. Functional class improved from $3.2 \pm 0.4$ to $1.7 \pm 0.7$ in the surviving patients $(p<0.01)$. Furthermore, left ventricular ejection fraction improved from $19.8 \% \pm 3 \%$ to $23.9 \% \pm$ $7.2 \%(p<0.01)$, and significant changes in stroke index, arterial pressure, pulmonary wedge pressure, and left ventricular stroke work index were also found at 6 months of follow-up. In the late postoperative period, the left ventricular ejection fraction tended to decrease and returned to preoperative levels at 5 years, whereas hemodynamic variables did not change significantly. Thus, despite the tendency of the left ventricular ejection fraction to decrease at late follow-up, the long-term course of these patients seems to be characterized by the maintenance of hemodynamic improvement. However, long-term survival after cardiomyoplasty is limited by the severity of the patient's condition before the operation. (J THORAC CARDIOVASC SURG 1995;109:353-63)
\end{abstract}

Luiz Felipe P. Moreira, MD (by invitation), Noedir A. G. Stolf, MD (by invitation), Edimar A. Bocchi, MD (by invitation), Fernando Bacal, MD (by invitation), Paulo M. Pêgo-Fernandes, MD (by invitation), Henry Abensur, MD (by invitation), José C. Meneghetti, MD (by invitation), and Adib D. Jatene, MD, São Paulo, Brazil

$\mathrm{D}_{\mathrm{y}}$ ynamic cardiomyoplasty has been proposed by several authors as an alternative to heart transplantation in the treatment of patients with severe

From the Instituto do Coração da Faculdade de Medicina da Universidade de São Paulo, São Paulo, Brazil.

Supported by grant 521/88/55 from Fundação E. J. Zerbini, São Paulo, Brazil.

Read at the Seventy-fourth Annual Meeting of The American Association for Thoracic Surgery, New York, N.Y., April 24-27, 1994.

Address for reprints: Luiz Felipe P. Moreira, MD, Divisão de Cirurgia, Instituto do Coração, Av. Dr. Enéas C. Aguiar, 44—São Paulo, SP, Brazil, CEP: 05403-000.

Copyright (C 1995 by Mosby-Year Book, Inc.

0022-5223/95 $\$ 3.00+0 \quad \mathbf{1 2 / 6 / 6 0 4 5 8}$ cardiomyopathies. ${ }^{1-9}$ Its primary objectives are to reverse congestive heart failure and to halt the progression of the underlying disease by the increase of ventricular pumping performance and by the reinforcement of ventricular walls.

Improvement in congestive heart failure and left ventricular function were demonstrated after cardiomyoplasty in patients with dilated or ischemic cardiomyopathies. ${ }^{1-9}$ Initial results also suggest that this procedure may improve long-term survival in patients with advanced heart failure. ${ }^{10}$ Nevertheless, potential limitations and risk factors of poor cardiomyoplasty outcome have been further reported. ${ }^{1-5,9}$ In addition, the absence of previous studies about long-term changes in left ventricular function 
after this surgical procedure has precluded definitive conclusions about its value.

The purpose of this report is to document the clinical and left ventricular function effects of dynamic cardiomyoplasty up to 5 years of follow-up in patients with advanced heart failure caused by idiopathic dilated cardiomyopathy. The preoperative factors influencing the outcome are also analyzed.

\section{Patients and methods}

Study population. From May 1988 to November 1993, 31 patients with idiopathic dilated cardiomyopathy were treated by dynamic cardiomyoplasty at the Heart Institute, University of São Paulo Medical School. Their ages ranged from 28 to 62 years (mean 48.2 years); 23 were men and eight were women. Twenty-four patients were in New York Heart Association functional class III and seven were in class IV despite maximal medical therapy with digitalis, diuretics, and angiotensin-converting enzyme inhibitors. They also had had an average of 2.6 hospitalizations for treatment of congestive heart failure in the year preceding the operation. Five of these patients had reversible cardiogenic shock with temporary use of intravenous inotropic drugs.

All patients had a left ventricular ejection fraction below $30 \%$, as documented at radioisotopic scintigraphy, and had high left ventricular filling pressures at heart catheterization (mean pulmonary wedge pressure or left ventricular end-diastolic pressure above $15 \mathrm{~mm} \mathrm{Hg}$ ). The absence of significant coronary artery compromise and diffuse, severe left ventricular dilatation were also demonstrated by heart catheterization in every patient. Mitral valve insufficiency was present in 24 patients, being moderate in five and mild in 19 patients.

Twenty-seven patients were in sinus rhythm and the remaining four were in atrial fibrillation. Holter monitor recordings also showed that 12 patients had more than 50 premature ventricular contractions per hour and that 15 patients had several episodes of nonsustained ventricular tachycardia. Standard spirometric study documented mild restrictive compromise of ventilatory function in 26 patients.

In the present investigation, patients receiving intravenous inotropic drugs were not candidates for cardiomyoplasty. Furthermore, important or severe valvular dysfunction, arrhythmias not controlled by medical therapy, and pulmonary or multiple organ diseases were also considered contraindications for this operation.

Medical or psychosocial contraindications to heart transplantation were present in most of these patients. All patients signed a special informed consent form according to the Scientific Committee of the Heart Institute.

Surgical technique. Dynamic cardiomyoplasty was performed without extracorporeal circulation by means of two separate incisions: a lateral approach for muscle flap dissection and a subsequent median sternotomy for cardiac access. Dissection and transposition of the left latissimus dorsi muscle followed the technique described by Carpentier and associates. ${ }^{11}$ The skeletal muscle flap was then wrapped around the ventricular surfaces and fixed directly to the myocardium by separated sutures, provid- ing a left posterior cardiocostal wrapping. ${ }^{6}$ Two intramuscular pacing electrodes (Medtronic SP 5528, Medtronic, Inc., Minneapolis, Minn.) were implanted in the skeletal muscle graft and an intramyocardial sensing lead (Medtronic SP 5548) was placed in either the right or left ventricle. The cardiomyostimulator Medtronic SP 1005 was used for muscle flap stimulation.

Anesthetic management of cardiomyoplasty included the systematic use of dopamine, dobutamine, and sodium nitroprusside, associated with the maintenance of adequate volume loading, to improve the hemodynamic profile and to stabilize arterial blood pressure even while the heart was being wrapped in muscle. ${ }^{12}$ The prophylactic use of intravenous inotropic support was continued for at least 1 week after the operation.

Muscle flap stimulation. Skeletal muscle flap stimulation started 2 weeks after the operation, and a progressive muscle conditioning protocol was followed. ${ }^{11}$ In the late postoperative period, the muscle flap was paced in 1:1 or 1:2 synchrony with the heart rate. Pulse train stimulation was used with a burst frequency of $30 \mathrm{~Hz}$ and was associated with supramaximal pulse amplitude values to avoid ineffective muscle flap contraction. The delay between the ventricular sensed event and the muscle burst was adjusted to provide an exact synchronization between muscle flap contraction and ventricular systole under echocardiographic monitoring. ${ }^{6}$

Follow-up protocol. Ventricular function was evaluated in all patients before the operation, at 6 months of follow-up, and annually until the fifth postoperative year by means of Doppler echocardiography, radioisotopic angiography, and right-sided heart catheterization.

Doppler echocardiography was performed with the Aloka SSD-730 and SSD-870 sector scans (Aloka Inc., Tokyo, Japan). A $2.5 \mathrm{MHz}$ transducer was used for $\mathrm{M}$-mode and two-dimensional studies, whereas a $2 \mathrm{MHz}$ transducer was used for Doppler determinations. The standard parameters of left ventricular function (left ventricular end-diastolic diameter, left ventricular endsystolic diameter, and left ventricular segmental wall shortening) were obtained by M-mode and two-dimensional echocardiograms. Left ventricular stroke volume and the degree of mitral valve insufficiency were determined by Doppler analysis.

Radioisotopic scintigraphy was obtained after intravenous injection of technetium $99 \mathrm{~m}$. Gated blood pool imaging was obtained in the left anterior oblique position with a Siemens model LEM+ camera (Siemens Corp., Union, N.J.). The images were analyzed in a Microvax model 3300 computer (Siemens): left and right ventricular ejection fractions were calculated.

For right-sided heart catheterization, a thermodilution balloon-tipped catheter was positioned in the pulmonary artery. Pressures were measured with a strain gauge transducer, whereas cardiac output was obtained by the thermodilution technique. Arterial pressures were measured by noninvasive standard means. Cardiac index, stroke index, left and right ventricular stroke work indexes, and vascular resistances were calculated by standard formulas.

Statistical analysis. Cumulative mortality rates over time were determined by the actuarial method, and 
differences between them were assessed by the log-rank test. Repeated-measures analysis of variance was used to compare data obtained before and after cardiomyoplasty. A $p$ value less than 0.05 was considered significant. Data are presented as mean plus or minus standard deviation, whereas survival rates are presented as mean plus or minus standard error of the mean.

So that the association of variables to survival could be assessed, univariate analysis was performed by means of the unpaired Student's $t$ test and Fisher's exact test. Variables with a $p$ value less than 0.10 in univariate analysis were entered into the Cox proportional-hazards regression model. Patients with less than 1 year of follow-up were excluded from this analysis and patients undergoing urgent heart transplantation after cardiomyoplasty were included as deaths.

\section{Results}

Immediate surgical results. No intraoperative or hospital deaths occurred in this series. Three patients required countershock for ventricular fibrillation while the heart was being wrapped with muscle.

The immediate postoperative course was uneventful in general. One patient had low cardiac output despite prophylactic pharmacologic support and required intraaortic balloon counterpulsation. A transient period of renal failure and mesenteric ischemia developed in another patient. Four patients had transitory atrial fibrillation or flutter and two patients had pulmonary complications.

Muscle flap ischemic compromise was monitored by variations in the serum level of creatine kinase enzyme, which showed a mean peak value of $946 \pm$ 582 IU. Four patients had peak values of creatine kinase greater than $1500 \mathrm{IU}$, and these patients had poor muscle flap contraction at the beginning of the muscle stimulation protocol.

During the muscle conditioning period one patient had progressive heart failure and successfully underwent urgent heart transplantation 42 days after the cardiomyoplasty. Another patient died at 2 months of severe pulmonary thromboembolism and infection after having been discharged from the hospital.

Long-term follow-up. Twenty-nine patients completed the muscle conditioning protocol, and they were followed up from 6 to 70 months (mean 25.8 months). In the first 19 patients, the muscle flap pacing was usually synchronized in a 1:1 mode with the heart rate. In the remaining 10 patients, the muscle flap was normally paced in a 1:2 mode.

At 6 months of follow-up, New York Heart Association functional class improved from $3.2 \pm$ 0.4 to $1.7 \pm 0.7(p<0.01)$. The number of hospi- talizations during the follow-up period significantly decreased to 0.6 per patient per year $(p<0.01)$.

On the other hand, 11 other patients died at long-term follow-up. The cause of death was progressive heart failure in seven patients, and four patients died suddenly. Progressive heart failure was associated with pulmonary complications in four patients and with renal failure, cerebral vascular accident, or myocarditis in the remaining three. The actuarial survival curve represented in Fig. 1 shows survivals of $86 \% \pm 6.4 \%$ at 1 year, $61.4 \% \pm 10 \%$ at 2 years, and $42.5 \% \pm 11.8 \%$ from 3 to 5 years of follow-up.

Seven of the surviving patients are currently in functional class I, eight in class II, and two in class III, some of them using fewer drugs or lower doses than they did in the preoperative period. Another patient who had progressive heart failure also underwent heart transplantation at 22 months of follow-up.

Univariate analysis of preoperative risk factors showed that functional class IV and elevated pulmonary vascular resistance were associated with unfavorable outcome (Table I). When multivariate analysis was performed, only preoperative functional class was identified as a significant predictor of survival $(p<0.01)$. The survival curves according to the preoperative functional class are depicted in Fig. 2.

Ventricular function evaluation. Data obtained by Doppler echocardiography are shown in Table II. Significant improvements of left ventricular wall shortening and left ventricular stroke volume were found at 6 months of follow-up. Otherwise, left ventricular wall shortening tended to decrease after the first year of follow-up, whereas left ventricular stroke volume maintained the improvement recorded at 6 months up to 5 years after cardiomyoplasty. Doppler echocardiographic studies also showed that the degree of mitral insufficiency did not change in the late follow-up period.

Right and left ventricular ejection fraction data obtained by radioisotopic scintigraphy are also depicted in Table II. Left ventricular ejection fraction improved early after the cardiomyoplasty from $19.8 \% \pm 3 \%$ to $23.9 \% \pm 7.2 \%(p<0.01)$. Nevertheless, this parameter also tended to decrease at late follow-up and returned to the preoperative levels at 5 years, as shown in Fig. 3.

The hemodynamic evaluation showed that significant improvements of arterial pressure, stroke index, and left ventricular stroke work index were 


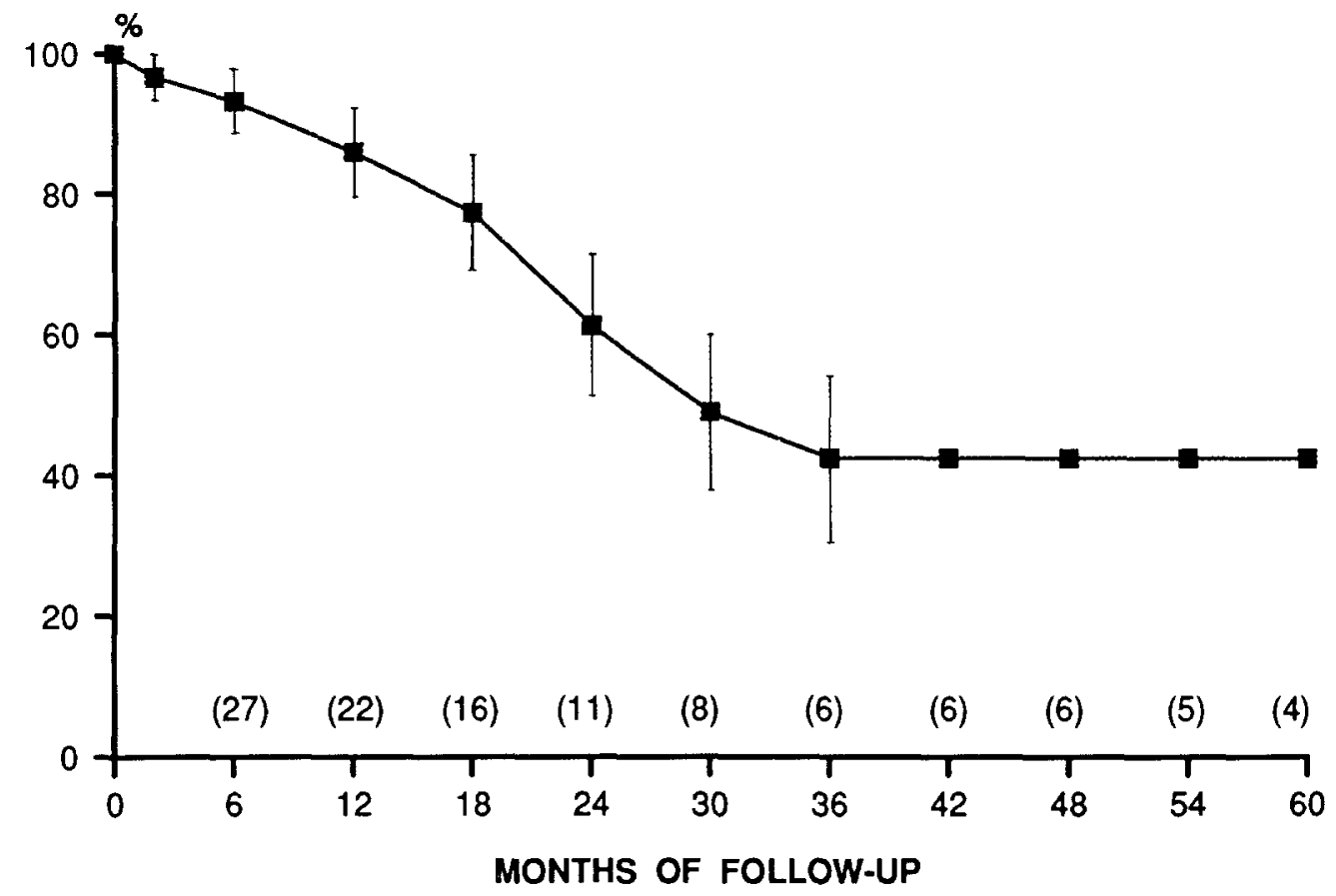

Fig. 1. Actuarial survival curve after dynamic cardiomyoplasty. Numbers in parentheses indicate patients who completed the follow-up period. Values are presented as mean \pm standard error of the mean.

Table I. Unifactorial analysis of factors associated with outcome

\begin{tabular}{lc}
\multicolumn{1}{c}{ Parameter } & Significance \\
\hline Functional class (NYHA) & $p=0.005$ \\
Pulmonary vascular resistance & $p=0.028$ \\
Mean pulmonary artery pressure & $p=0.096$ \\
Systolic index & $p=0.144$ \\
Systemic vascular resistance & $p=0.165$ \\
Cardiothoracic ratio & $p=0.168$ \\
Mean right atrial pressure & $p=0.179$ \\
Mean pulmonary wedge pressure & $p=0.309$ \\
Left ventricular end-diastolic pressure & $p=0.358$ \\
Cardiac index & $p=0.381$ \\
Left ventricular diastolic diameter & $p=0.485$ \\
Right ventricular ejection fraction & $p=0.579$ \\
\hline
\end{tabular}

NYHA, New York Heart Association.

associated with significant decreases in pulmonary artery pressure and pulmonary wedge pressure at 6 months of follow-up (Table III). In the late postoperative period, this study showed that the hemodynamic benefits of cardiomyoplasty persisted for up to 5 years of follow-up. Mean pulmonary wedge pressures are depicted in Fig. 4, and left ventricular stroke work index calculations are presented in Fig. 5.

\section{Discussion}

The present investigation showed that the longterm course of patients with idiopathic dilated cardiomyopathy who underwent cardiomyoplasty was characterized by the maintenance of the hemodynamic improvement noted early after the operation. This improvement was maintained despite the tendency of left ventricular ejection fraction to decrease to preoperative levels at late follow-up.

Otherwise, long-term survival after cardiomyoplasty was limited by the severity of the patient's condition before the operation. Thus this procedure should be used in patients with some degree of myocardial functional reserve and without severe pulmonary hypertension. In this regard, cardiomyoplasty was performed in this series without hospital mortality and it did not preclude heart transplantation, as also described by others. ${ }^{1,13}$ Furthermore, the shortage of donors and the increasing number of candidates requiring urgent treatment have decreased the number of heart transplants for outpatient candidates. ${ }^{14,15}$ This situation justifies the use of alternative surgical options for patients with moderate or severe cardiomyopathies who cannot have a reasonable quality of life and prognosis on 


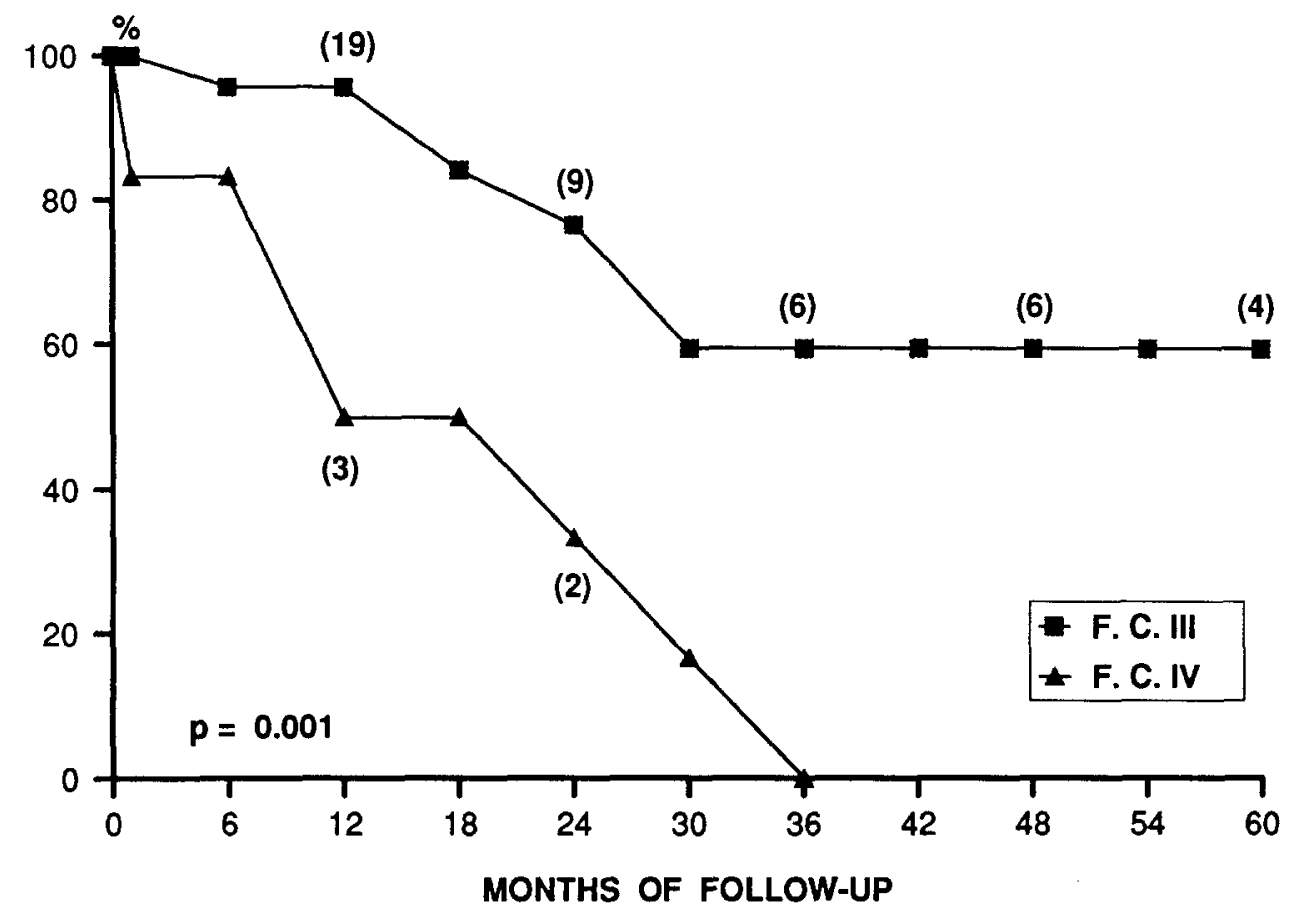

Fig. 2. Actuarial survival curves after dynamic cardiomyoplasty for patients operated in New York Heart Association functional classes III (F.C. III) or IV (F.C.IV). Numbers in parentheses indicate patients who completed the follow-up period.

Table II. Noninvasive evaluation of ventricular function

\begin{tabular}{lccccccc}
\hline & \multicolumn{7}{c}{ Follow-up } \\
\cline { 3 - 8 } & $\begin{array}{c}\text { Preop } \\
(n=28)\end{array}$ & $\begin{array}{c}6 \text { mo } \\
(n=28)\end{array}$ & $\begin{array}{c}12 \text { mo } \\
(n=22)\end{array}$ & $\begin{array}{c}24 \text { mo } \\
(n=11)\end{array}$ & $\begin{array}{c}36 \text { mo } \\
(n=6)\end{array}$ & $\begin{array}{c}48 \text { mo } \\
(n=6)\end{array}$ & $\begin{array}{c}60 \text { mo } \\
(n=4)\end{array}$ \\
\hline Doppler echocardiography & & & & & & \\
LVDD (mm) & $73.1 \pm 6.1$ & $73.6 \pm 7.7$ & $73.8 \pm 7.7$ & $73.7 \pm 3.1$ & $75.7 \pm 5.1$ & $76.3 \pm 4.7$ & $77.5 \pm 6.7$ \\
LVSWS (\%) & $11.9 \pm 1.9$ & $15.5 \pm 2.9^{*}$ & $14.7 \pm 3.5^{*}$ & $13.8 \pm 3.3$ & $13.2 \pm 1.9$ & $11.3 \pm 2$ & $11.7 \pm 1.9$ \\
LVSV (ml) & $27.3 \pm 9.1$ & $33.5 \pm 11.4^{*}$ & $37.8 \pm 11.8^{*}$ & $36.4 \pm 12^{*}$ & $39.3 \pm 10.8$ & $35 \pm 15.1$ & $34 \pm 13.1$ \\
Radioisotopic scintigraphy & & & & & & $20.8 \pm 3$ & $20.5 \pm 3.6$ \\
LVEF (\%) & $19.7 \pm 3.3$ & $23.9 \pm 6.6^{*}$ & $23.3 \pm 6.5^{*}$ & $23.3 \pm 6.2$ & $22.5 \pm 3.6$ & $20.8 \pm 3.5$ \\
RVEF (\%) & $25.8 \pm 7.7$ & $24.9 \pm 8.6$ & $26.5 \pm 8.1^{*}$ & $27.5 \pm 7.3^{*}$ & $26.5 \pm 5.4$ & $26.2 \pm 4.4$ & $25.5 \pm 5.7$ \\
\hline
\end{tabular}

$L V D D$, Left ventricular diastolic diameter; $L V S W S$, left ventricular segmental wall shortening; $L V S V$, left ventricular stroke volume; $L V E F$, left ventricular ejection fraction; $R V E F$, right ventricular ejection fraction. Numbers in parentheses indicate the patients evaluated at each period.

${ }^{*} p<0.05$ in relation to preoperative data of matched patients.

medical therapy for heart failure. This population currently represents more than 2200 patients awaiting transplantation in the United States alone, with only a $4 \%$ chance of receiving a heart each month. ${ }^{15}$

Ventricular function changes. Several authors have demonstrated the improvement of left ventricular ejection fraction after dynamic cardiomyoplasty. $1,4,8,9$ In a recent report, our group ${ }^{16}$ showed that this improvement occurs from a global modification of left ventricular regional wall motion and may be associated with significant changes in the left ventricular shape. Also important is the fact that the same study ${ }^{16}$ and other reports ${ }^{1,6}$ demonstrated the decrease of left ventricular ejection fraction when the cardiomyostimulator was turned off for several hours, which ruled out the possibility of a placebo effect.

Besides ejection fraction changes, selection of patients with severe impairment of ventricular function and pulmonary hypertension in the current investigation was advantageous for showing the beneficial hemodynamic effects of cardiomyoplasty, as 


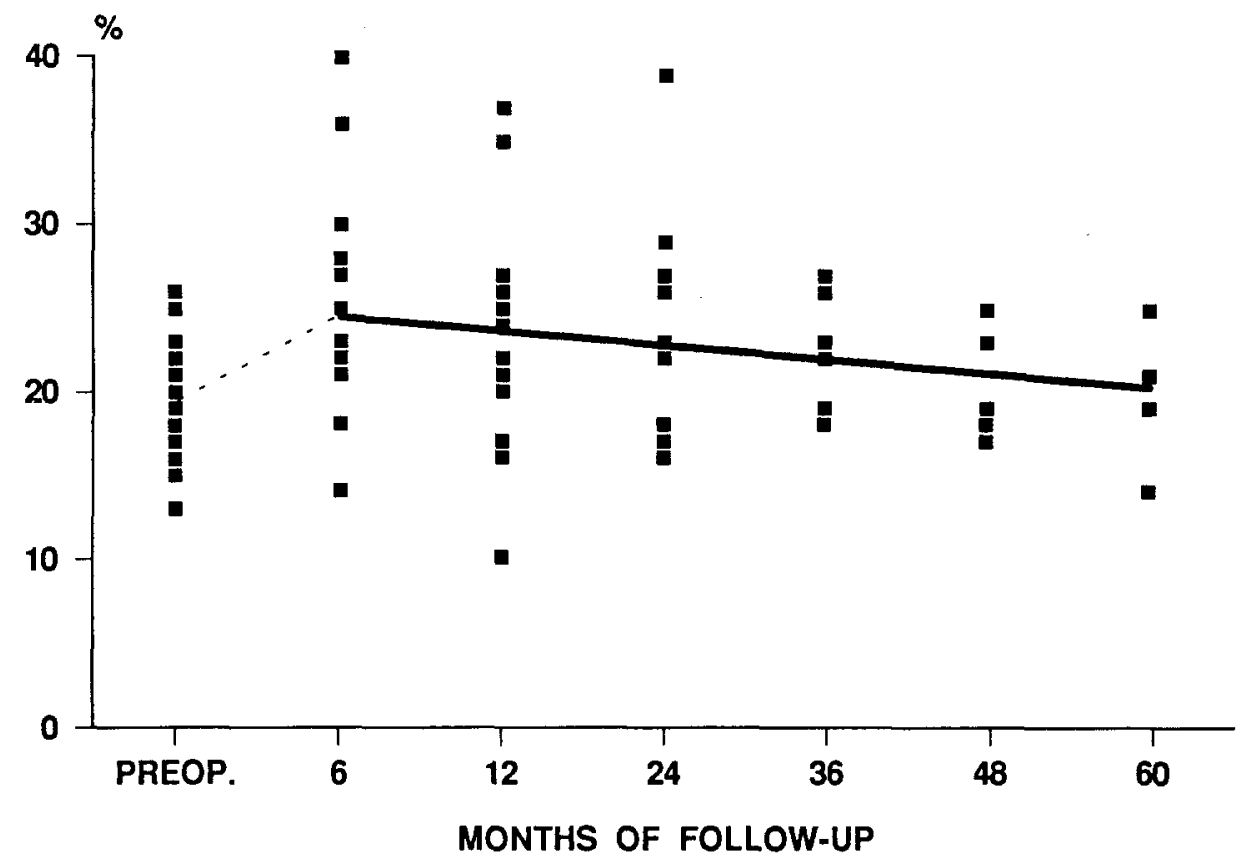

Fig. 3. Plots of left ventricular ejection fraction values obtained by radioisotopic scintigraphy in the preoperative period and up to 60 months after dynamic cardiomyoplasty. The solid line indicates the postoperative trend.

Table III. Hemodynamic evaluation

\begin{tabular}{|c|c|c|c|c|c|c|c|}
\hline & \multirow[b]{2}{*}{$\begin{array}{c}\text { Preop } \\
(n=28)\end{array}$} & \multicolumn{6}{|c|}{ Follow-up } \\
\hline & & $\begin{array}{c}6 m o \\
(n=28)\end{array}$ & $\begin{array}{c}12 \text { mo } \\
(n=22)\end{array}$ & $\begin{array}{c}24 m o \\
(n=11)\end{array}$ & $\begin{array}{c}36 \text { mo } \\
(n=6)\end{array}$ & $\begin{array}{c}48 m o \\
(n=6)\end{array}$ & $\begin{array}{c}60 \mathrm{mo} \\
(n=4)\end{array}$ \\
\hline MRAP (mm Hg) & $9 \pm 3.6$ & $9.1 \pm 5.3$ & $8.9 \pm 5.2$ & $9.1 \pm 6.3$ & $6.7 \pm 5.6$ & $7.2 \pm 5.6$ & $8 \pm 4.7$ \\
\hline MPAP (mm Hg) & $36.1 \pm 11.2$ & $29.8 \pm 9.6^{*}$ & $30.3 \pm 9.4^{*}$ & $29.8 \pm 11.1^{*}$ & $24 \pm 6.8$ & $28.2 \pm 8.4$ & $30.5 \pm 7.5$ \\
\hline MPWP (mm Hg) & $24.7 \pm 6.3$ & $18.5 \pm 6^{*}$ & $18.2 \pm 5.8^{*}$ & $17.9 \pm 7.7^{*}$ & $15.2 \pm 3.2^{*}$ & $15.5 \pm 6.1$ & $19 \pm 6.6$ \\
\hline MAP (mm Hg) & $86.1 \pm 7.7$ & $93.1 \pm 10.6^{*}$ & $91.7 \pm 11.1$ & $93.2 \pm 8.8^{*}$ & $96.2 \pm 12.4^{*}$ & $98.8 \pm 15.3$ & $90.3 \pm 7.9$ \\
\hline $\mathrm{CI}\left(\mathrm{L} / \mathrm{min} \cdot \mathrm{m}^{2}\right)$ & $1.94 \pm 0.31$ & $2.02 \pm 0.4$ & $2.04 \pm 0.44$ & $1.97 \pm 0.26$ & $2.3 \pm 0.3$ & $2.17 \pm 0.26$ & $2.18 \pm 0.2$ \\
\hline SI $\left(\mathrm{ml} / \mathrm{m}^{2}\right)$ & $20.6 \pm 3.1$ & $24 \pm 5.3^{*}$ & $24.7 \pm 6.8^{*}$ & $25 \pm 6.7^{*}$ & $28.9 \pm 4.4^{*}$ & $27 \pm 6.8$ & $25.7 \pm 4.9$ \\
\hline RVSWI $\left(\mathrm{gm} \cdot \mathrm{m} / \mathrm{m}^{2}\right)$ & $7.4 \pm 2.1$ & $6.9 \pm 2.8$ & $7 \pm 3.6$ & $6.9 \pm 2.6$ & $6.7 \pm 1.4$ & $7.3 \pm 0.7$ & $7.7 \pm 0.7$ \\
\hline LVSWI $\left(\mathrm{gm} \cdot \mathrm{m} / \mathrm{m}^{2}\right)$ & $17.5 \pm 5.2$ & $25.5 \pm 9.1^{*}$ & $25.6 \pm 9.4^{*}$ & $25.4 \pm 7^{*}$ & $30.9 \pm 9.5^{*}$ & $29.7 \pm 10.9 *$ & $25.5 \pm 8.5$ \\
\hline PVR (dyne $\cdot \mathrm{sec} \cdot \mathrm{cm}^{-5}$ ) & $288 \pm 166$ & $271 \pm 96$ & $282 \pm 136$ & $267 \pm 94$ & $183 \pm 107$ & $288 \pm 165$ & $236 \pm 70$ \\
\hline SVR $\left(\right.$ dyne $\cdot \mathrm{sec} \cdot \mathrm{cm}^{-5}$ ) & $1862 \pm 258$ & $1962 \pm 329$ & $1882 \pm 393$ & $1930 \pm 365$ & $1748 \pm 166$ & $1838 \pm 305$ & $1638 \pm 141$ \\
\hline
\end{tabular}

$M R A P$, Mean right atrial pressure; $M P A P$, mean pulmonary artery pressure; $M P W P$, mean pulmonary wedge pressure; $M A P$, mean arterial pressure; $C I$, cardiac index; SI, systolic index; RVSWI, right ventricular stroke work index; $L V S W$, left ventricular stroke work index; $P V R$, pulmonary vascular resistance; $S V R$, systemic vascular resistance. Numbers in parenthesis indicate the patients evaluated at each period.

${ }^{*} p<0.05$ in relation to preoperative data of matched patients.

previously published..$^{6,9}$ More precisely, although left ventricular stroke volume and cardiac output augmentation at rest were modest after this operation, the impact of cardiomyoplasty on circulatory function could be better evaluated by its significant influence on left ventricular stroke work and pulmonary pressures. In addition, more evident changes in cardiac output and global circulatory function dur- ing exercise have been clinically shown after this procedure. $^{17}$

The enhancement of ventricular function after cardiomyoplasty involves, furthermore, modifications in the mechanical properties of the failing heart. Another study showed that the effects of synchronous skeletal muscle flap contraction on left ventricular systolic function also include the in- 


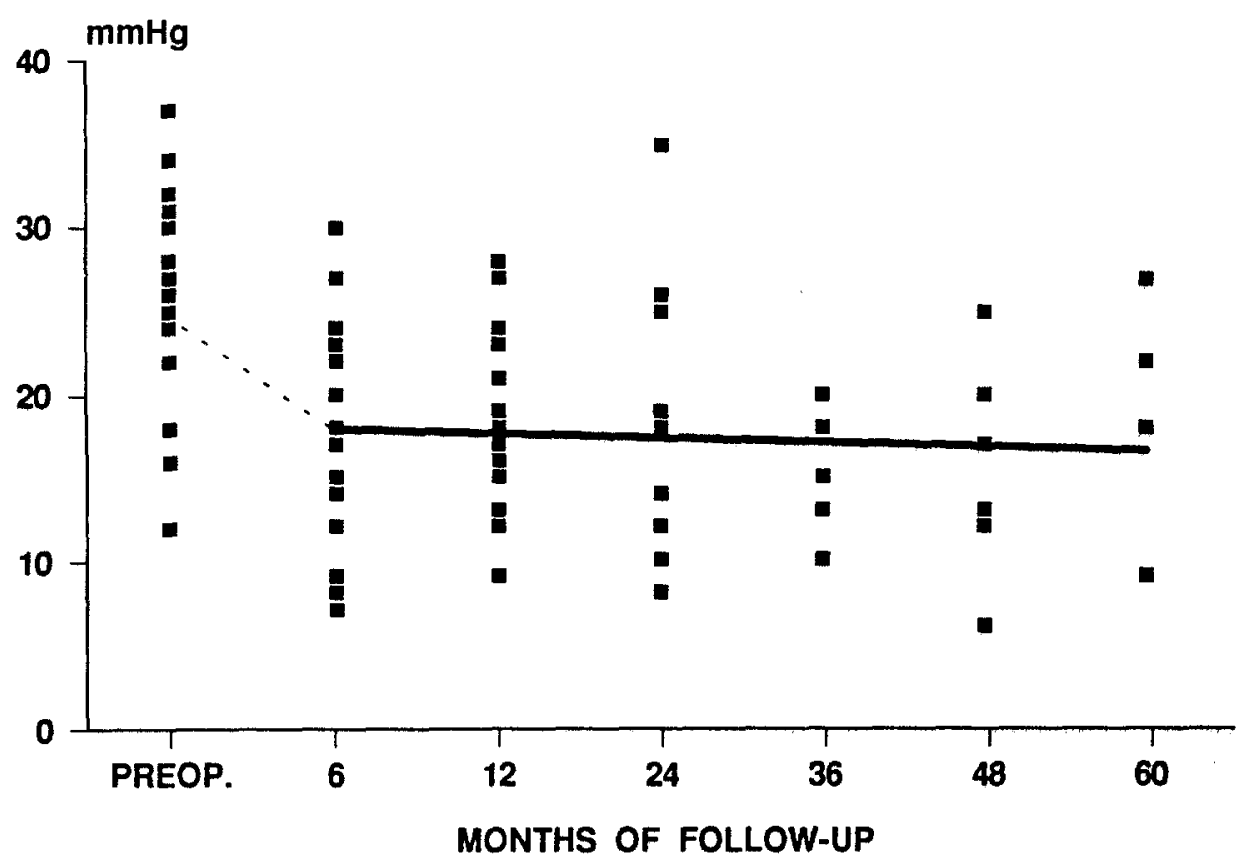

Fig. 4. Plots of mean pulmonary wedge pressure values obtained by hemodynamic evaluation in the preoperative period and up to 60 months after dynamic cardiomyoplasty. The solid line indicates the postoperative trend.

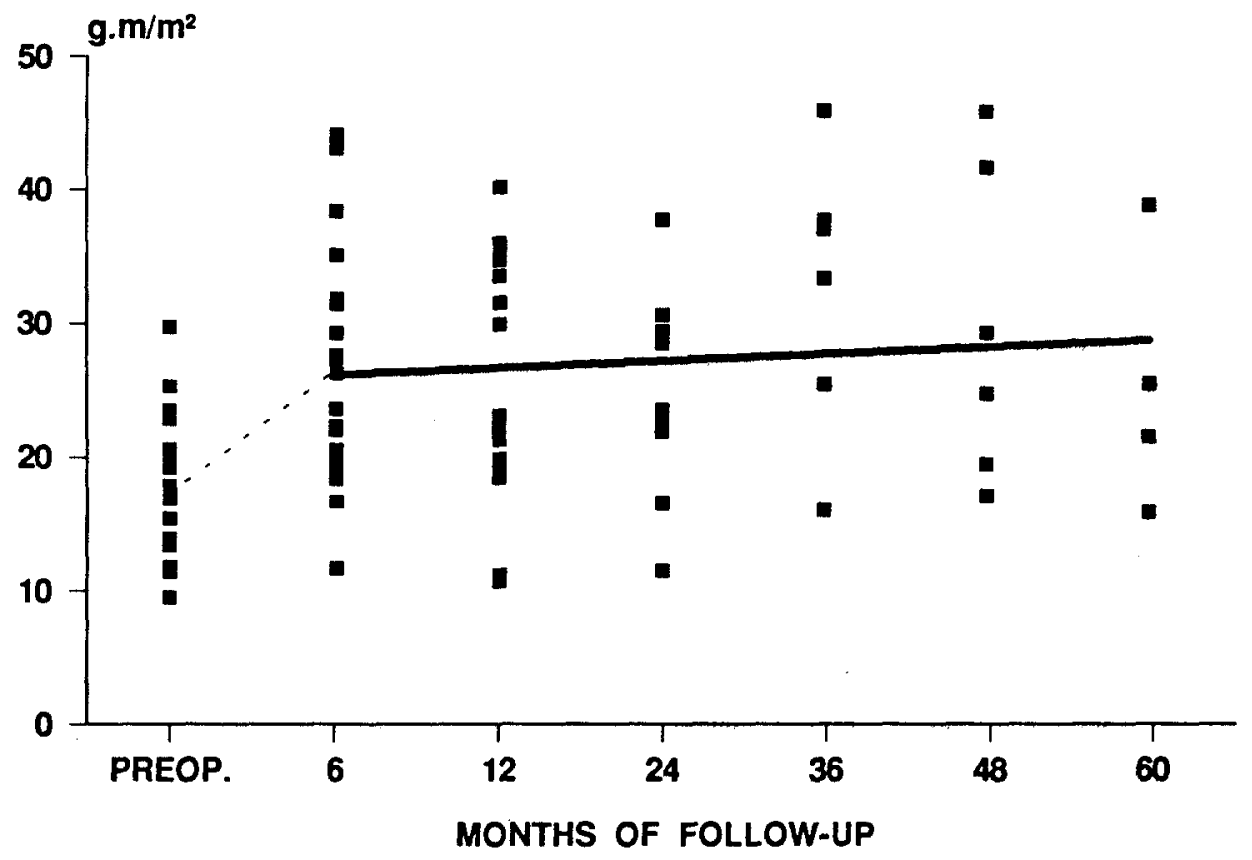

Fig. 5. Plots of left ventricular stroke work index values obtained by hemodynamic evaluation in the preoperative period and up to 60 months after dynamic cardiomyoplasty. The solid line indicates the postoperative trend. 
crease of the maximal elastance and the decrease of the wall stress. ${ }^{18}$ Regarding the diastolic function, decreases in end-diastolic pressure, stress, and chamber and muscle stiffness were also noticed. Because left ventricular wall stress is an important determinant of myocardial oxygen consumption, the reduction in wall stress can reduce myocardial oxygen demand, as demonstrated experimentally. ${ }^{19,20}$ Accordingly, in a dilated, failing left ventricle, the primary pathophysiologic abnormalities ${ }^{21,22}$ are partially corrected, and slow progression of the underlying cardiomyopathy can result from this mechanism.

Maintenance of hemodynamic improvement for up to 5 years after cardiomyoplasty in the present study seems to indicate that heart failure had stopped progressing in the surviving patients. However, the tendency of left ventricular ejection fraction to decrease at late follow-up suggests that the effects of this procedure on heart failure may be limited to a mid-term period. Stabilization in the process of ventricular dilatation and maintenance of normal hemodynamic parameters for more than 3 years after this operation were also reported by Carpentier and colleagues, ${ }^{1}$ but their experience included mainly patients with ischemic cardiomyopathy.

Functional improvement. In addition to changes in ventricular function, the improvement in functional class after cardiomyoplasty in this series agrees with the general clinical experience with this procedure..$^{1-9}$ Most of our patients were in functional class I or II, and a significant decrease in the number of hospitalizations per patient per year was also found.

The functional benefits of cardiomyoplasty also include a decrease in the patient's limitations for physical activities, food and sleep patterns, and social activities, as we previously demonstrated by quality of life assessment. ${ }^{23}$ Improvement in exercise capacity was similarly shown by the treadmill exercise test both in Carpentier's experience ${ }^{1}$ and in our experience. $^{24}$

Effects on survival. Despite the functional improvement revealed in this investigation, late mortality after cardiomyoplasty was high. Heart failure progressed, especially in patients with important preoperative compromise or with evidence of skeletal muscle flap ischemia in the immediate postoperative period. ${ }^{25}$ Sudden cardiac death was also observed, but it was less prevalent than that described in the literature-approximately $20 \%$ to
$30 \%$ per year in patients with chronic heart failure. ${ }^{26}$ Nevertheless, this complication is probably not affected by cardiomyoplasty and still needs effective treatment.

It is important to emphasize that the current indications for cardiomyoplasty included mainly patients at high risk of dying within 1 year, because they did not essentially differ from patients awaiting heart transplantation. ${ }^{27}$ Moreover, we ${ }^{10}$ already reported that the survival after this operation was much more favorable than the survival of a comparable, but not randomized, group of patients maintained on medical therapy. In that study, patients undergoing to cardiomyoplasty had a survival of $65 \%$ at 2 years of follow-up, whereas the 2 -year survival was only $27 \%$ in the medically treated group.

Also of interest is the fact that the mortality after cardiomyoplasty in this series was significantly higher in patients who were in functional class IV or who had elevated pulmonary vascular resistance, which suggests that the degree of cardiac function impairment before the operation plays an important role in the long-term outcome. Other studies also showed the impact of these factors on cardiomyoplasty results, ${ }^{1,5}$ and additional risk factors influencing perioperative and late mortality after this procedure have been similarly reported. ${ }^{1,25}$ Furnary and associates ${ }^{4}$ observed a significant influence of right ventricular ejection fraction on early mortality after cardiomyoplasty, and Carpentier and colleagues ${ }^{1}$ described the importance of surgical factors and of atrial fibrillation for hospital and late outcomes.

Application of these findings to patient selection criteria may improve the benefits of cardiomyoplasty. In this regard, patients operated on in functional class III in this series had survivals of $76 \%$ at 2 years and of $59 \%$ from 3 to 5 years of follow-up. These values positively contrast with the recent reports of heart failure treatment with angiotensinconverting enzyme inhibitors or vasodilators, which showed a survival probability of only $40 \%$ in 5 years for patients in class III. ${ }^{28,29}$ Furthermore, the survival expectation may be even worse in the presence of persistent abnormalities of hemodynamic profile, as documented in the preoperative period in most of the patients in this investigation. ${ }^{30-32}$

Postoperative muscle flap performance. Besides preoperative factors, cardiomyoplasty outcome may also be influenced by technical aspects and by skeletal muscle flap response to electrical stimula- 
tion. The use of right ${ }^{8}$ or left latissimus dorsi muscle and the influence of the extent of the wrap around the heart ${ }^{2}$ remain controversial.

Also important are the possibility of perioperative ischemic compromise of the muscle flap ${ }^{9}$ and the fact that the distal part of the latissimus dorsi muscle may eventually become fibrotic and atrophic. ${ }^{33}$ These region-specific changes were found in clinical cases by necropsy studies and by magnetic resonance images, ${ }^{34}$ but their influence on long-term performance of the muscle flap remains unclear. The possible relationship between these findings and the late modifications of left ventricular ejection fraction in the present study suggests, however, the necessity of further investigations regarding the prevention of muscle flap ischemia and the optimal stimulation protocols for transformation and assistance.

Study limitations. This report describes the longterm effects of cardiomyoplasty on left ventricular function. Nevertheless, the number of individuals was not sufficiently large for statistical validation of data at 4 and 5 years of follow-up. The decreasing number of patients with time was inevitable, especially as a result of the late deaths.

Regarding the clinical outcome of cardiomyoplasty, this study strongly suggested that this operation may improve long-term survival of patients with severe cardiomyopathies who remain in functional class III despite maximal medical therapy. However, this finding was based only on data obtained in the literature. Also important was the fact that the analysis of preoperative factors associated with outcome was limited by the number of patients in this series, indicating that it will require further validation.

\section{Conclusions}

Despite many controversies about this surgical procedure, the present experience with dynamic cardiomyoplasty demonstrated that it improves left ventricular function, ameliorates congestive heart failure, and is a low-risk operation in patients with idiopathic dilated cardiomyopathy. Furthermore, the long-term course of the surviving patients after cardiomyoplasty seems to be characterized by the stabilization of the underlying disease for up to 5 years of follow-up.

Although long-term survival after this operation may be limited by the severity of the patient's preoperative condition, this procedure seems otherwise to be a promising alternative to the treatment of advanced heart failure in patients in functional class III or reversible class IV who also have mild or moderate pulmonary hypertension. Further studies are needed, however, to improve technical aspects and to confirm these observations with data from a larger, randomized clinical trial, comparing cardiomyoplasty and medical therapy outcomes.

We are grateful for the kind collaboration of Mrs. Creusa M. Roveri Del Bó, consultant statistician.

\section{REFERENCES}

1. Carpentier A, Chachques JC, Acar C, et al. Dynamic cardiomyoplasty at seven years. J THORAC CARDIOVASC SURG 1993;106:42-53.

2. Chiu RC-J, Odim JNK, Burgess JH, The McGill Cardiomyoplasty Group. Responses to dynamic cardiomyoplasty for idiopathic dilated cardiomyopathy. Am J Cardiol 1993;72:475-9.

3. Delahaye F, Jegaden O, Montagna P, et al. Latissimus dorsi cardiomyoplasty in severe congestive heart failure: the Lyon experience. J Cardiac Surg 1991; 6(Suppl):106-12.

4. Furnary AP, Magovern JA, Christlieb IY, Orie JE, Simpson KA, Magovern GJ. Clinical cardiomyoplasty: preoperative factors associated with outcome. Ann Thorac Surg 1992;54:1139-43.

5. Grandjean PA, Austin L, Chan S, Terpstra B, Bourgeois I. Dynamic cardiomyoplasty: clinical follow-up results. J Cardiac Surg 1991;6(Suppl):80-8.

6. Jatene $\mathrm{AD}$, Moreira LFP, Stolf NAG, et al. Left ventricular function changes after cardiomyoplasty in patients with dilated cardiomyopathy. J THORAC CARDIOVASC SURG 1991;102:132-9.

7. Lorusso R, Zogno M, La Canna G, et al. Dynamic cardiomyoplasty as an effective therapy for dilated cardiomyopathy. J Cardiac Surg 1993;8:177-83.

8. Magovern JA, Park SE, Cmolik BL, Trumble DR, Christlieb IY, Magovern GJ. Early effects of right latissimus dorsi cardiomyoplasty on left ventricular function. Circulation 1993;88(Suppl):II298-303.

9. Moreira LFP, Bocchi EA, Stolf NAG, Pileggi F, Jatene AD. Current expectations in dynamic cardiomyoplasty. Ann Thorac Surg 1993;55:299-303.

10. Moreira LFP, Seferian P Jr, Bocchi EA, et al. Survival improvement with dynamic cardiomyoplasty in patients with dilated cardiomyopathy. Circulation 1991; 84(Suppl):III296-302.

11. Carpentier A, Chachques JC, Grandjean PA, eds. Cardiomyoplasty. Mt. Kisco, N.Y.: Futura, 1991.

12. Auler JOC Jr, Moreira LFP, Carvalho MJ, Stolf NAG, Amaral RVG, Jatene AD. Anesthetic management of patients undergoing cardiomyoplasty. Anesthesiology 1992;77:379-81.

13. Jegaden O, Delahaye F, Montagna P, et al. Cardio- 
myoplasty does not preclude heart transplantation. Ann Thorac Surg 1992;53:875-81.

14. McMannus RP, O'Hair DP, Beitzinger JM, et al. Patients who die awaiting heart transplantation. J Heart Lung Transplant 1993;12:159-72.

15. Stevenson LW, Warner SL, Steimle AE, et al. The impending crisis awaiting cardiac transplantation: modeling a solution based on selection. Circulation 1994;89:450-7.

16. Bocchi EA, Moreira LFP, Moraes AV, et al. Effects of dynamic cardiomyoplasty on regional wall motion, ejection fraction and geometry of left ventricle. Circulation 1992;86(Suppl):II231-5.

17. Bocchi EA, Moreira LFP, Bellotti G, et al. Hemodynamic study during upright isotonic exercise before and six months after dynamic cardiomyoplasty for idiopathic dilated cardiomyopathy or Chagas' disease. Am J Cardiol 1991;67:213-4.

18. Bellotti G, Moraes AV, Bocchi EA, et al. Late effects of cardiomyoplasty on left ventricular mechanics and diastolic filling. Circulation 1993;88(Suppl):II304-8.

19. Kawaguchi O, Goto Y, Futaki S, Ohgoshi Y, Yaku H, Suga $H$. Mechanical enhancement and myocardial oxygen saving by synchronized dynamic left ventricular compression. J Thorac CARDIOvasc SuRg 1992; 103:573-81.

20. Lee KF, Dignan RJ, Parmar JM, et al. Effects of dynamic cardiomyoplasty on left ventricular performance and myocardial mechanics in dilated cardiomyopathy. J Thorac CARDIOVASC SURg 1991;102:12431.

21. Katz AM. Cardiomyopathy of overload: a major determinant of prognosis in congestive heart failure. $\mathrm{N}$ Engl J Med 1990;322:100-10.

22. Packer M. Pathophysiology of chronic heart failure. Lancet 1992;340:88-93.

23. Borghetti-Maio SA, Romano BW, Bocchi EA, et al. Quality of life after cardiomyoplasty. J Heart Lung Transplant 1994;13:271-5.

24. Moreira LFP, Stolf NAG, Jatene AD. Benefits of cardiomyoplasty for dilated cardiomyopathy. Semin Thorac Cardiovasc Surg 1991;3:140-4.

25. Bocchi EA, Bellotti G, Moreira LFP, et al. Prognostic indicators of one-year outcome after cardiomyoplasty for idiopathic dilated cardiomyopathy. Am J Cardiol 1994;73:604-8.

26. Larsen L, Markham J, Haffajee CI. Sudden death in idiopathic dilated cardiomyopathy: role of ventricular arrhythmias. PACE 1993;16:1051-9.

27. Vagelos R, Fowler MB. Selection of patients for cardiac transplantation. Cardiol Clin 1990;8:23-38.

28. The SOLVD Investigators. Effect of enalapril on survival in patients with reduced left ventricular ejection fractions and congestive heart failure. $\mathrm{N}$ Engl $\mathrm{J}$ Med 1991;325:293-302.

29. Cohn JN, Johnson G, Ziesche S, et al. A comparison of enalapril with hydralazine-isosorbide dinitrate in the treatment of chronic congestive heart failure. $\mathrm{N}$ Engl J Med 1991;325:303-10.

30. Morley D, Brozena SC. Assessing risk by hemodynamic profile in patients awaiting cardiac transplantation. Am J Cardiol 1994;73:379-83.

31. Stevenson LW, Tillisch JH, Hamilton MA, et al. Importance of hemodynamic response to therapy in predicting survival with ejection fraction $\leq 20 \%$ secondary to ischemic or nonischemic cardiomyopathy. Am J Cardiol 1990;66:1348-54.

32. Steimle AE, Stevenson LW, Fonarow GC, Hamilton MA, Morigushi JD. Prediction of improvement in recent onset cardiomyopathy after referral for heart transplantation. J Am Coll Cardiol 1994;23:553-9.

33. Kratz JM, Johnson WS, Mukherjee RM, Hu J, Crawford FA, Spinale FG. The relation between latissimus dorsi skeletal muscle structure and contractile function after cardiomyoplasty. J THORAC CardiovasC SURG 1994;107:868-78.

34. Kalil R, Bocchi EA, Weiss R, et al. MRI evaluation of chronic morphological changes in the latissimus dorsi cardiomyoplasty. Circulation 1994;90(Suppl):II107-11.

\section{Discussion}

Dr. George J. Magovern (Pittsburgh, Pa.). We have performed the cardiomyoplasty procedure on approximately 38 patients at Allegheny General Hospital. Our first five patients were out of protocol, but they were good-risk patients. Three of the five are still alive with dramatically improved ejection fractions.

We then began using the Food and Drug Administration protocol, and we had a $30 \%$ operative mortality. I believe nine of the patients died of arrhythmia, with one current survivor. They were mostly ischemic patients who had been candidates for transplantation.

The third group, comprising 15 patients, was also in a Food and Drug Administration protocol. Our results at 2 years parallel the results reported by Dr. Moreira's group.

Dr. Moreira, did you do all your cases through a left thoracotomy or did you use a combined approach? The duration of the operative procedure is probably critical.

Have you compared any primary cardiomyopathy cases with ischemic cardiomyopathy cases? We believe it is critical to preserve the muscle, and we are considering a 2-week delay in the procedure, after the muscle is freed, to allow for the development of collaterals.

We have noticed that if we stop the stimulation for say a 2-week period and then restart it, the ejection fraction improves. Have you ever turned the pacer off and let the muscle wrap rest and relax?

Our first group of patients had no operative mortality, and four of five survived. It is unique that in two of the patients who are still living the ejection fraction has remained significantly improved.

Dr. Moreira: Dr. Magovern, thank you very much for your comments. Regarding your first question, we used two incisions, a lateral approach for muscle flap dissection and a subsequent sternotomy for the heart procedure. 
This operation takes about 8 hours, and I do not think that its length is a critical problem.

The sudden cardiac deaths in this series occurred especially in patients with important arrhythmias. I believe this problem may be even more important in ischemic patients.

Regarding muscle flap preservation, I think we need to study better how to decrease the skeletal muscle ischemic compromise in the cardiomyoplasty procedure. Furthermore, we need to evaluate better stimulation patterns to achieve a more powerful muscle flap contraction in the long term, without late compromise of the skeletal muscle function and integrity. Regarding the muscle flap synchronization ratio, we used both the $2: 1$ and 1:1 modes. Most of our patients had better results with the 1:1 mode. Otherwise, I believe that the idea of stopping the muscle stimulation for some time at night may be beneficial and may need to be investigated.

Dr. John M. Kratz (Charleston, S.C.). Your data seem to suggest some late problem with the whole procedure and a tailing off of benefits. Our laboratory work and that of others have also suggested later problems with the muscle, such as fibrosis. Did you have the opportunity to do any autopsies on patients dying in the late postoperative period? What was the muscle like at that time?

Dr. Moreira. We performed some autopsies and also studied some patients by means of magnet resonance imaging while the cardiomyostimulator was being replaced. These patients were evaluated between 2 and 5 years of follow-up. Most of then had some degree of fibrosis and fatty tissue infiltration in the latissimus dorsi muscle flap. On the other hand, it is important to emphasize that patients having magnet resonance imaging were also subjected to left ventricular function evaluation with the cardiomyostimulator turned on and off. These studies showed a significant decrease of left ventricular ejection fraction in some patients when the myostimulator was turned off, even in the presence of muscle flap fibrosis and fat tissue infiltration.

\section{Availability of JouRNAL back issues}

As a service to our subscribers, copies of back issues of THE Journal of THORACIC AND CARdiovascular SURGERY for the preceding 5 years are maintained and are available for purchase from the publisher, Mosby-Year Book, Inc., at a cost of $\$ 12.00$ per issue. The following quantity discounts are available: $25 \%$ off on quantities of 12 to 23 , and one third off on quantities of 24 or more. Please write to Mosby-Year Book, Inc., Subscription Services, 11830 Westline Industrial Drive, St. Louis MO 63146-3318, or call (800)453-4351 or (314)453-4351 for information on availability of particular issues. If unavailable from the publisher, photocopies of complete issues are available from University Microfilms International, 300 N. Zeeb Rd., Ann Arbor, MI 48106, (313)761-4700. 